\title{
MULTIPLE ABSCESSES OF THE SPLEEN
}

\author{
Wg Cdr KL SAMPATH KUMAR * , Wg Cdr J ZUTSHI ${ }^{+}$, \\ Sqn Ldr A ALAM"
}

MJAFI 2000; 56 : 266-267

KEY WORDS: Abscess; Spleen.

\section{Introduction}

$\mathbf{S}$ plenic abscess is an unusual entity. In the last 30 years, in a busy hospital like the Johns Hopkins Hospital, only 11 patients have been treated for clinically overt splenic abscess [11. The clinical presentation is nonspecific and diagnosis is usually delayed. Prior to 1970 , diagnosis was by exclusion, and delay in treatment was frequent. But the diagnosis has been made more frequently in recent years due to the increasing number of living immunocompromised patients and to the use of more sophisticated radiological diagnosis techniques like splenic scintiscans, ultrasonography and computerised axial tomography [2].

The predisposing factors for splenic abscess are preceding pyogenic infection, haematologic disorders such as sickle cell disease, trauma and contiguous disease in the pancreas.

Although splenectomy has been advocated as the treatment of choice for splenic abscess, certain patients may be treated successfully with appropriate antibiotics and ultrasound guided simple drainage, preserving spleen [3].

Here, in this article, a case of multiple abscesses in the spleen, treated successfully with antibiotics and ultrasound guided percutanicuius unaillage is reported upon.

\section{Case Report}

A 27-year-old male patient was admitted to the hospital with history of fever of moderate to high degree of 10-days duration associated with off and on rigors. Prior to this he was treated with Tab Ciproflaxacin by a private practitioner without any relief.

At the time of admission, the patient gave no history of cough or breathlessness. There was no history of trauma to the left lower chest nor to the left upper quadrant of abdomen. There was history of loss of appetite and loss of weight of $2 \mathrm{~kg}$ in the last 10 days. Bowels and bladder were normal. On examination the patient was found to be febrile, the temperature being 39 degree celsius. The patient had pallor. Examination of abdomen revealed just palpable spleen below the left costal margin on deep inspiration. The spleen was non tender. Rest of the examination including the respiratory system was unremarkable. The patient was investigated. Urine examination was normal. Blood haemoglobin was $10 \mathrm{gm}$ percent with TLC $9000 /$ cu.mm. and polymorphs 40 , lymphocytes 55 , eosinophils 03 , and monocytes 02 percent. Blood for Widal test was strongly positive and when repeated there was rise in titre. The blood culture was found to be sterile. The radiograph of chest was reported to be normal except for minimal raised left dome of diaphragm. The patient was put on oral Ciprofloxacin and antipyretics. However the patient continued to be febrile and on the third day of hospitalisation, he complained of left upper abdominal pain and was found to have left upper quadrant (LUQ) abdominal tenderness. Radiograph of chest was repeated. It revealed evidence of left basal pneumonitis with pleural effusion and raised left hemidiaphragm (Fig-1). In view of above findings, ultrasound examination of abdomen was carried out. It revealed enlarged spleen with four anechoic areas with thick blood like echogenic contents, the largest of these measuring $74 \times 55 \mathrm{~mm}$. The patient was subjected to Computerised Axial Tomography (CAT) of abdomen. This revealed multiple abscesses in the spleen. The largest abscess measured approximately $80 \times 59 \mathrm{~mm}$ (Fig-2). Although splenectomy has been advocated as the treatment of choice for splenic abscess $[3,4]$, this patient was put on broad spectrum antibiotics in the form of Inj. Ciprofloxacin $200 \mathrm{mg}$ IV 8th hrly, Inj. Gentamicin $80 \mathrm{mg}$ IV 8th hrly and Inj.Flagyl $500 \mathrm{mg}$ IV 8th hrly. Then the patient was subjected to ultrasound guided percutaneous drainage of the abscess. About $80 \mathrm{ml}$ of 'anchovy sauce' type of pus was drained from the largest abscess of the spleen. Since the other three abscesses were small they were not subjected to aspiration.

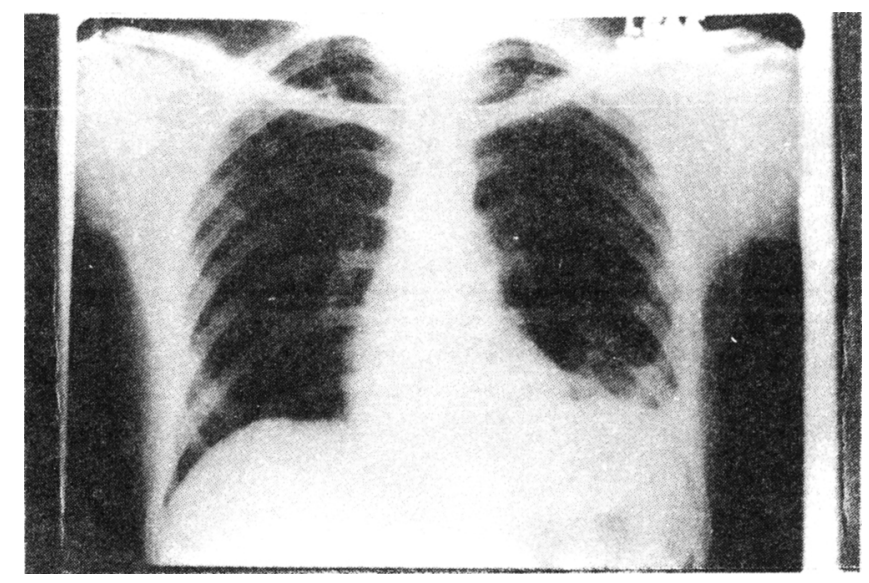

Fig. 1: Radiograph of chest showing evidence of left basal pneumonitis with left pleural effusion and raised left hemidiaphragm

\footnotetext{
Classified Specialist (Surgery), ${ }^{+}$Medical Specialist, ${ }^{\#}$ Classified Specialist (Radiodiagnosis), 9 Air Force Hospital, Halwara-AD 141106.
} 


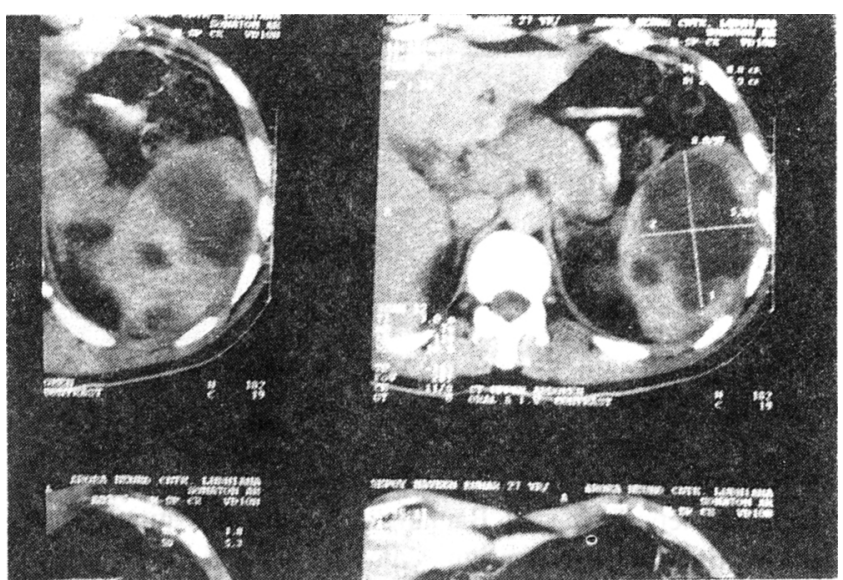

Fig. 2: CT scan of abdomen showing multiple abscesses in the spleen, the largest measuring approximately $80 \times 59 \mathrm{~mm}$

Though the microscopic examination of the pus revealed the clusters of Gram positive cocci, the culture of the pus was found to be sterile. Following the aspiration, the patient recovered very rapidly. The patient became afebrile, his appetite improved and showed good general improvement within a week's time. Ultrasound examination was repeated after one week, which revealed good healing of the abscesses. Hence splenectomy was not contemplated. The antibiotics were also stopped after 10 days. The patient recovered well and was asymptomatic on subsequent periodic reviews.

\section{Discussion}

Earlier most splenic abscesses were multiple, small and clinically silent lesions found incidentally on autopsy and occurring as a terminal manifestation of uncontrolled infection elsewhere [5]. Clinically important splenic abscesses are generally solitary and arise from (1) systemic bacteremia originating in another site, such as endocarditis or salmonellosis (2) infection, probably by the haematogenous route, of a spleen damaged by bland infarction (as occurs in haemoglobinopathies, especially sickle cell trait or sickle cell disease), trauma, penetrating or blunt (with superinfection of a subcapsular haematoma) or other diseases (malaria, hydatid cysts); or (3) extension from a contiguous focus of infection, such as Subphrenic abscess [6]. However, in this case, the patient was not sufferring from any haemoglobinopathy as revealed subsequently by electrophoresis, nor he gave any history of trauma nor he had any subphrenic pathology. Most probably, this patient must have had systemic bacteremia such as salmonellosis resulting in splenic abscess. The blood culture was sterile because of antibiotic therapy before coming to the hospital. However positive Widal test was indicative of salmonellosis. The onset is typically subacute and the major features arc fever and the left upper quadrant (LUQ) abdominal pain. Here in this case also, the patient presented with the history of fever and developed typically the left upper quadrant tenderness. Typically the radiographic findings are elevation of the left hemidiaphragm and left pleural effusion as shown in this case. However the ultrasound scan and CT scan are more reliable diagnostic tests as in this case. It has become clear that since 1978, splenic abscess is diagnosed earlier in its presentation due to the widespread use of improved imaging techniques. The diagnosis of splenic abscess, however, is still often considered due to its rarity and the presence of predisposing conditions which obscure i. clinical presentation [7]. However, in this case though there was no obvious predisposing cause, in rewe uf left upper quandrant (LUQ) abdominal tenderness. ulluasonography and CT scan were carried out which clinched the diagnosis. Though splenectomy remains the traditional treatment, ultrasound guided percutaneous drainage was done in this case with success.

In conclusion. splenic abscess is an uncommon disease. However with the aid of new diagnostic tools, it is possible to diagnose this entity quite early and if treated adequately with broad spectrum antibiotics and ultrasound guided percutaneous drainage spleen can be preserved in selected patients.

\section{REFERENCES}

1. Sarr MG, Zuidema GD. Splenic abscess-presentation, diagnosis, and treatment. Surgery 1982; 92(3):480-3.

2. Caslowitz PL, Labs JD, Fishman EK, Siegelmann SS, Ressell $\mathrm{H}$. The changing spectrum of splenic abscess. Clin Imaging 1989;13(3):201-7.

3. Faught WE, Gilbertson JJ, Nelson EW. Splenic abscess; Presentation, treatment options and results. Am J Surg 1989:158(6):612-4.

4. Ooi LL, Leong SS. Splenic abscesses from 1987 to 1995. Am J Surg 1997;174(1):87-93.

5. Hirschmann JV. Localised infections and Abscesses. In: Braunwald E, Isselbacher KJ, Petersdorf RG, Wilson JD, Martin JB, Fauci AS, eds. Harrison's Principles of Internal Medicine. 11th ed. Newyork, McGraw Hill Book Company 1987;478-85.

6. Sheldon GF, Croom III RD, Meyer AA. The Spleen. In: Sabiston DC Jr, ed. Textbook of Surgery. 13th ed. Philadelphia, WB Saunders Company 1986;1203-29.

7. Nelken N, Ignatius J, Skinner M, Christensen N. Changing clinical spectrum of splenic abscess. A multicenter study and reivew of the literature. Am J Surg 1987;154(1):27-34. 